\title{
A geometric representation method using independent coordinates of the same control points
}

\author{
Zhenwei Wang ${ }^{1}$,Fang Fan ${ }^{2}$,Shuangyin Liu ${ }^{3}$ \\ ${ }^{1,2,3}$ School of Astronautics and Aeronautics, University of Electronic Science \& Technology of China, \\ Chengdu, 611731, PR China. \\ 1wangjanvey@163.com, ${ }^{2} 13007574791 @ 163 . c o m$
}

Keywords: shape representation, independent coordinates, Bézier curve or surface patch, equivalent parameter.

Abstract: Bézier curve is one of typical nonlinear splines, which is of importance for geometric representation, such as edge, contour. Generally, all control points are dealt with based on the same blending functions, regardless of the effect of every independent coordinates on geometric shape. Therefore, current representation lacks flexibility and controllability for complex shape. In this paper, we propose an equivalent parameter representation method using the independent coordinates of control points. Because all control point coordinates are used independently, geometric shape representation becomes more flexible and controllable. Particularly, different geometric shapes can be constructed by the same control points. Modeling experiments show that this proposed method is feasible and reasonable.

\section{Introduction}

Bézier curve possesses some advantages on nonlinear representation. It has brought more conveniences to complex shape representation. Hoffmann et al.[1] pointed out that the objective of solid modeling is to represent, manipulate and reason about three-dimensional shape of physical objects. Jüttler et al. [2] proposed Hermite interpolation algorithm for piecewise polynomial surfaces with rational offsets. Segall [3] discussed some mathematical and computer modelling of neural networks. In order to improve the uniformity of isoperimetric curves, Zhang et al.[4] presented the existence and numerical modelling of vortex rings with elliptic boundaries. Young et al.[5] adopted Bézier with even degree to solve offset approximation. Venkata et al.[6] gave parameters optimization algorithm of selected casting processes. Meanwhile, Orest et al.[7] discussed three-dimensional graphics representation of planar regions bounded by cubic Bézier curves. With aim of improvement on bound derivatives, Boumediene[8] proposed a new method for the modelling and stabilization of a nonlinear hybrid system of elasticity. In this paper, we propose an equivalent parameter geometric shape representation using independent coordinate of the same control points, which causes the reduction of modeling flexibility and controllability. The experiments show that using independent coordinates enables to expand modeling ability.

\section{Construction of Bézier Curve Using Independent Coordinates}

Generally, a cubic Bézier curve segment is expressed as Eq.1. The expression of variable $\boldsymbol{b}^{3}(u)$ can be calculated through blending functions. The coordinates $x, y$ and $z$ time the same blending function for any control point, as well as all control points. Generally, the parametric variable $u$ $(0 \leq u \leq 1)$ is used to determine any point position in curve segment. In order to represent a subcurve segment, we introduce two parametric variables $\lambda(0 \leq \lambda \leq 1)$ and $\gamma(0 \leq \gamma \leq 1)$ to refer to the position of 
start point and end point. Start point position and end point position are expressed as $c(\lambda)$ and $c(\gamma)$. Consequently, a subcurve segment is defined by four control points $\mathrm{c}(\lambda), P_{11}, P_{12}$ and $\mathrm{c}(\gamma)$.

$$
\mathbf{c}(u)=\stackrel{+}{\mathbf{t}(u) \cdot \mathbf{m}} \cdot \mathbf{p}=\mathbf{b}^{3}(u) \cdot \mathbf{p} \quad u \in\left[\begin{array}{ll}
0 & 1
\end{array}\right]
$$

This subcurve segment can be expressed as Eq.2, where the variable $P_{11}=\left[\mathrm{c}(\lambda), P_{7}, P_{9}, P_{3}\right]^{\mathrm{T}}$. Similarly, we can express the subcurve segment defined by the control points $P_{0}, P_{4}, P_{5}$ and c $(\lambda)$. Those control points are rewritten as the matrix variable $\mathbf{p}_{s}=\left(P_{0}, P_{4}, P_{5}, \mathbf{c}(\lambda)\right)^{T}$. Thus, the variable $\mathbf{p}_{s}$ is expressed as Eq.3 with corresponding to the matrix variable p, where the transformation matrix $\mathbf{b}=\left(\mathbf{b}^{0}(\lambda), \mathbf{b}^{1}(\lambda), \mathbf{b}^{2}(\lambda), \mathbf{b}^{3}(\lambda)\right)^{T}$. The transformation matrix $\mathbf{b}$ is a lower triangle matrix. From the view of geometric relationship, the transformation $\mathbf{b}$ represents the subset of a given curve segment at the beginning parametric variable $u=0$.

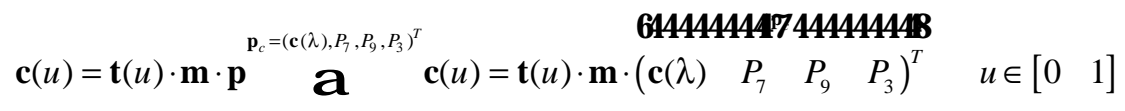

$$
\begin{aligned}
& \left(\begin{array}{c}
P_{0} \\
P_{4} \\
P_{5} \\
\mathbf{c}(\lambda)
\end{array}\right)=\left[\begin{array}{cccc}
1 & 0 & 0 & 0 \\
1-\lambda & \lambda & 0 & 0 \\
(1-\lambda)^{2} & 2(1-\lambda) \lambda & \lambda^{2} & 0 \\
(1-\lambda)^{3} & 3 \lambda(1-\lambda)^{2} & 3 \lambda^{2}(1-\lambda) & \lambda^{3}
\end{array}\right]\left(\begin{array}{l}
P_{0} \\
P_{1} \\
P_{2} \\
P_{3}
\end{array}\right) \text { a }\left(\begin{array}{c}
P_{0} \\
P_{4} \\
P_{5} \\
\mathbf{c}(\lambda)
\end{array}\right)=\left[\begin{array}{cccc}
b_{0}^{0}(\lambda) & 0 & 0 & 0 \\
b_{0}^{1}(\lambda) & b_{1}^{1}(\lambda) & 0 & 0 \\
b_{0}^{2}(\lambda) & b_{1}^{2}(\lambda) & b_{2}^{2}(\lambda) & 0 \\
b_{0}^{3}(\lambda) & b_{1}^{3}(\lambda) & b_{2}^{3}(\lambda) & b_{3}^{3}(\lambda)
\end{array}\right]\left(\begin{array}{c}
P_{0} \\
P_{1} \\
P_{2} \\
P_{3}
\end{array}\right)
\end{aligned}
$$

Next, we can represent the subcurve segment defined by the control points $\mathrm{c}(\lambda), P_{11}, P_{12}$ and $\mathrm{c}(\gamma)$. Similarly, this subcurve segment is a subset of the subcurve segment defined by the control points control points $\mathrm{c}(\lambda), P_{7}, P_{9}$ and $P_{3}$. Since both curves have the same beginning point $\mathrm{c}(\lambda)$, the transformation matrix $\mathbf{b}$ is the connection matrix between the variables $\mathbf{p}_{\mathrm{t}}$ and $\mathbf{p}_{\mathrm{c}}$. Thus, a problem is to keep the equivalent-parameter property for both curves. Generally, both variables $\lambda$ and $\gamma$ are not equal to zero. Therefore, we define the parametric variable $\varepsilon=\frac{\gamma-\lambda}{1-\lambda}(0 \leq \lambda \leq \gamma \leq 1)$. When the parameter variable $\lambda$ is given, $\gamma$ will vary from $\lambda$ and 1 . Thus, the range of parametric variable $\varepsilon$ is $\varepsilon \in[0,1]$. In Eq.3, we respectively substitute the variables $\mathbf{p}$ and $\mathbf{p}_{\mathrm{s}}$ into the variables $\mathbf{p}_{c}$ and $\mathbf{p}_{\mathrm{t}}$. Consequently, the transformation expression can be obtained with corresponding to the variables $\mathbf{p}_{c}$ to $\mathbf{p}_{\mathrm{t}}$, shown as Eq.4. Finally, a cubic Bézier subcurve is obtained by combining Eq.3 and Eq.4, shown as Eq.5.

$$
\begin{aligned}
& \begin{array}{l}
\left.\left(\begin{array}{c}
P_{0} \\
P_{4} \\
P_{5} \\
\mathbf{c}(\lambda)
\end{array}\right)=\left[\begin{array}{cccc}
b_{0}^{0}(\lambda) & 0 & 0 & 0 \\
b_{0}^{1}(\lambda) & b_{1}^{1}(\lambda) & 0 & 0 \\
b_{0}^{2}(\lambda) & b_{1}^{2}(\lambda) & b_{2}^{2}(\lambda) & 0 \\
b_{0}^{3}(\lambda) & b_{1}^{3}(\lambda) & b_{2}^{3}(\lambda) & b_{3}^{3}(\lambda)
\end{array}\right]\left(\begin{array}{c}
P_{0} \\
P_{1} \\
P_{2} \\
P_{3}
\end{array}\right) \begin{array}{l}
p_{p_{a}} \mathbf{p}_{\mathbf{p}_{t}} \\
\lambda a \varepsilon \\
\mathbf{a} \\
\mathbf{a} \\
\mathbf{c}(\lambda) \\
P_{11} \\
P_{12} \\
\mathbf{c}(\varepsilon)
\end{array}\right)=\left[\begin{array}{cccc}
b_{0}^{0}(\varepsilon) & 0 & 0 & 0 \\
b_{0}^{1}(\varepsilon) & b_{1}^{1}(\varepsilon) & 0 & 0 \\
b_{0}^{2}(\varepsilon) & b_{1}^{2}(\varepsilon) & b_{2}^{2}(\varepsilon) & 0 \\
b_{0}^{3}(\varepsilon) & b_{1}^{3}(\varepsilon) & b_{2}^{3}(\varepsilon) & b_{3}^{3}(\varepsilon)
\end{array}\right]\left(\begin{array}{c}
\mathbf{c}(\lambda) \\
P_{7} \\
P_{9} \\
P_{3}
\end{array}\right)
\end{array} \\
& \mathbf{c}(u)=k \cdot \mathbf{b}^{3}(u) \cdot\left[\begin{array}{cccc}
b_{0}^{0}(\varepsilon) & 0 & 0 & 0 \\
b_{0}^{1}(\varepsilon) & b_{1}^{1}(\varepsilon) & 0 & 0 \\
b_{0}^{2}(\varepsilon) & b_{1}^{2}(\varepsilon) & b_{2}^{2}(\varepsilon) & 0 \\
b_{0}^{3}(\varepsilon) & b_{1}^{3}(\varepsilon) & b_{2}^{3}(\varepsilon) & b_{3}^{3}(\varepsilon)
\end{array}\right] \cdot\left[\begin{array}{cccc}
b_{0}^{3}(\lambda) & b_{1}^{3}(\lambda) & b_{2}^{3}(\lambda) & b_{3}^{3}(\lambda) \\
0 & b_{0}^{2}(\lambda) & b_{1}^{2}(\lambda) & b_{2}^{2}(\lambda) \\
0 & 0 & b_{0}^{1}(\lambda) & b_{1}^{1}(\lambda) \\
0 & 0 & 0 & b_{0}^{0}(\lambda)
\end{array}\right]\left(\begin{array}{c}
P_{0} \\
P_{1} \\
P_{2} \\
P_{3}
\end{array}\right)
\end{aligned}
$$




\section{Experiments on Variables of Curves}

Figure 2 shows some 2D and 3D subcurves under conditions of different variable $k$. Both subfigures have the same variables $\lambda=0.2$ and $\varepsilon=0.75(\gamma=0.8)$. It proves that the same control points can generate different cubic Bézier curve segments. Particularly, subcurve shape is controlled by the variables $\lambda, \varepsilon$ and $k$ for given control points. Therefore, those variables make the curve representation easier and more flexible.

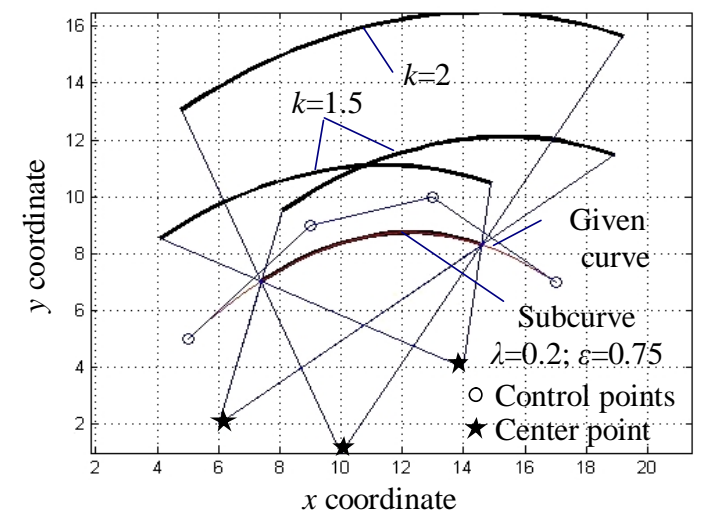

(a) Transformations of two-dimensional shape

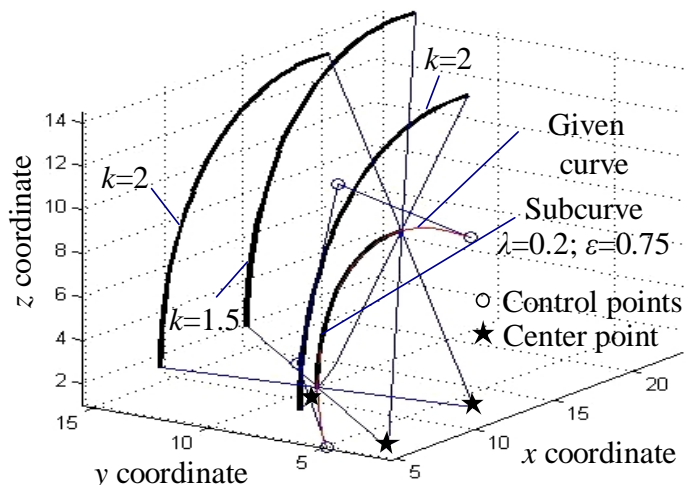

(b) Transformations of three-dimensional shape

Fig.1 Transformation examples of cubic Bézier subcurve segment

The variables $\lambda, \varepsilon$ and $k$ are respectively decomposed into $\varepsilon_{x}, \lambda_{x}, k_{x}$ in the direction of $x$ coordinate, and $\varepsilon_{y}, \lambda_{y}, k_{y}$ in the direction of $y$ coordinate, and $\varepsilon_{z}, \lambda_{z}, k_{z}$ in the direction of $z$ coordinate. Figure 3 shows some curves under conditions of different coordinate variables $k_{x}, k_{y}$ and $k_{z}$. Other coordinate variables are set to $\lambda_{x}=\lambda_{x}=\lambda_{x}=0.2$ and $\varepsilon_{x}=\varepsilon_{y}=\varepsilon_{z}=0.75$. From both subfigures, it can be found that the variables $k_{x}, k_{y}$ and $k_{z}$ respectively scales the subcurve segment in the direction of $x, y$ and $z$ coordinate. Therefore, the variables $k_{x}, k_{y}$ and $k_{z}$ will independently generate different Bézier subcurve segments.

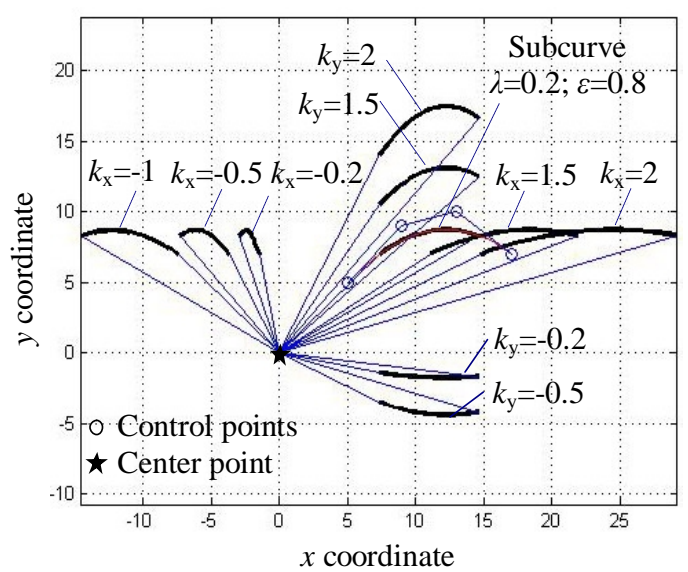

(a) Two dimension curves with corresponding to $\boldsymbol{k}$

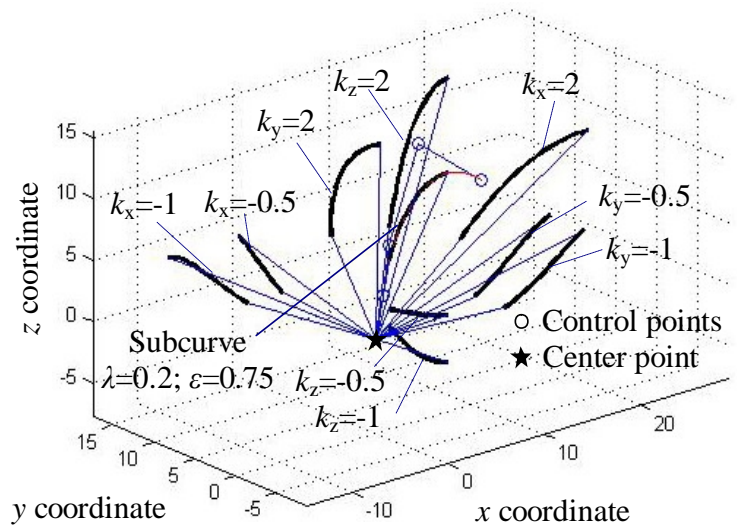

(b) Three dimension curve with corresponding to $\boldsymbol{k}$

Fig.2 Curve transformations with variety of parameter $\boldsymbol{k}$

As an example, Figure 4 illustrates two Bézier curve graphs. Firstly, Fig.4(a) shows two curve sets under conditions of different variable $k_{x}$ and $k_{y}$. The first curve set is drawn under conditions of $k_{x}=1$ and the second curve set is plotted under conditions of $k_{x}=-1$. Since all subcurve segments have the same boundaries, this geometric shape can be used to express computation boundaries. Secondly, Fig.4(b) shows a branch model under conditions of the constant variable $\boldsymbol{k}=(1,1)$. From this subfigure, it is found that the variables $u_{x}$ and $u_{y}$ respectively conduct the subcurve selection in 
the direction $x$ and $y$ coordinates. Therefore, geometric shape can be generated based on coordinate variables $k_{x}, k_{y}, u_{x}$ and $u_{y}$.
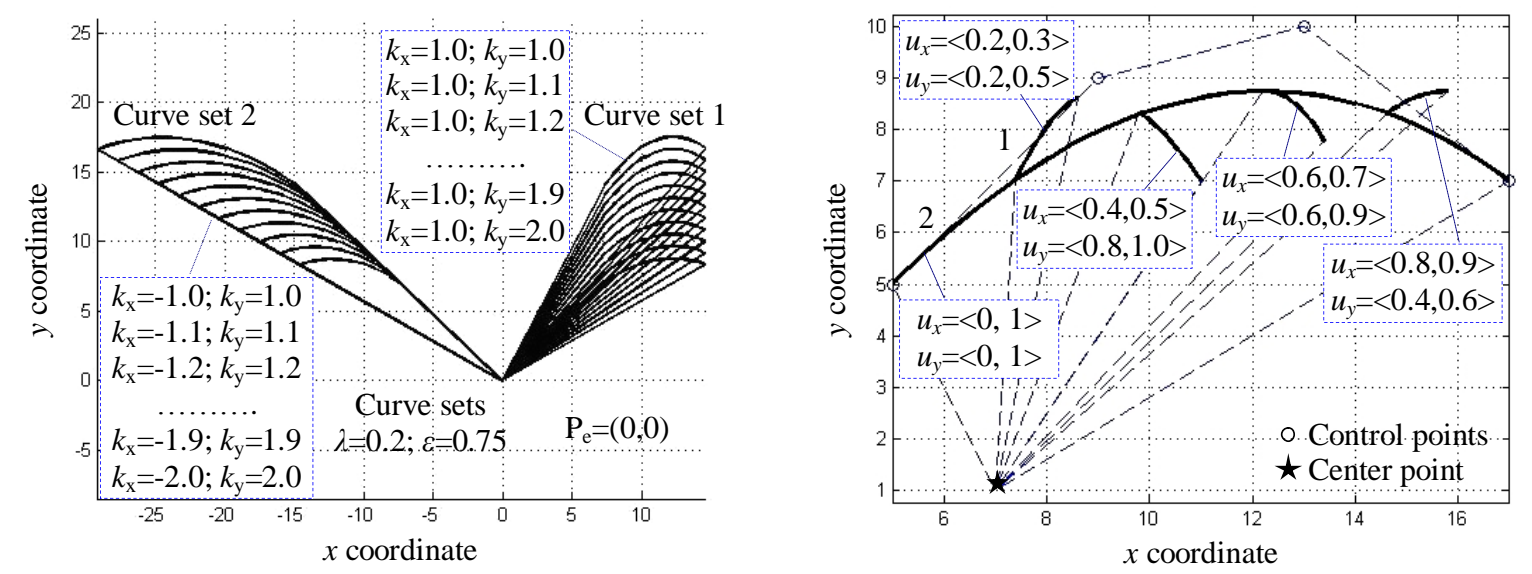

Fig.3 Two Bézier subcurve examples

\section{Acknowledgments}

This work was financially supported by the Fundamental Research Funds for the Central Universities (No.ZYGX2014J095) and the National Natural Science Foundation of China (No.51005040)

\section{References}

[1]Christoph M. Hoffmann, Jaroslaw R. Rossignac. A road map to solid modeling [J]. IEEE transaction on visualization and computer graphics, 1996, 2(1), 1-10.

[2]Jüttler B, Sampoli ML. Hermite interpolation by piecewise polynomial surfaces with rational offsets [J]. Compute Aided Geometric design 2000;17:361-385.

[3]R. S. Segall. Some mathematical and computer modelling of neural networks[J]. Applied mathematical modelling, 19 (7), 1995: 386-399.

[4]Yan Zhang, Lonut Danaila. Existence and numerical modelling of vortex rings with elliptic boudaries [J].Applied mathematical modelling, 37 (7), 2013: 4809-4824.

[5]Young Joon Ahn, Christoph Hoffmann. Circle approximation using LN Bézier curves of even degree and itsapplication [J]. Journal of Mathematical Analysis and Applications, 410 (2014) 257-266.

[6]R.Venkata Rao, V.D.Kalyankar, G.Waghmare. Parameters optimization of selected casting processes using teaching-learning-based optimization algorithm[J]. Applied mathematical modelling, 38 (23), 2014: 5592-5608.

[7]Orest Shardt, John C. Bowman. Surface parameterization of nonsimply connected planar Bézier regions[J]. Computer-Aided Design 44 (2012) 484.e1-484.e10.

[8]Boumediene Chentouf. Modelling and stabilization of a nonlinear hybrid system of elasticity [J]. Applied mathematical modeling 39 (2), 2015: 621-629. 